\title{
Endocervicalcolposcopy: an exploratory method complementary to colposcopy
}

\author{
Abstract \\ Objective: The purpose of this study is to show a new exploratory technique that used as \\ complement of the colposcopy, can increase his diagnostic capacity enables exploration \\ of the endocervical canal, and can transform unsatisfactory colposcopy into satisfactory \\ colposcopy.
}

Materials and methods: This is a descriptive study that shows the results of 136 endocervicalcolposcopy, performed after standard colposcopy. The procedures were conducted from January 5, 2010 to June 30, 2014, and the follow-up period consisted of least two years to detect the persistence or recurrence of lesions, and to verify the presence of lesions not detected by colposcopy or endocervicalcolposcopy. Lesions that went undetected by normal colposcopy but were detected by endocervicalcolposcopy are described, as is the effectiveness of this method to transform unsatisfactory colposcopy into satisfactory colposcopy.

Results: Endocervicalcolposcopy detected 17 lesions that had not been detected by standard colposcopy (12.5\% of all patients), converted 53 of the 57 unsatisfactory colposcopies $(92.98 \%)$ into satisfactory colposcopy. The procedure was useful in $41.17 \%$ of all cases. Only 8 endocervicalcolposcopies were unsatisfactory $(5.88 \%)$.

Commentary: Endocervicalcolposcopy is an exploratory method that is simple and easy to perform. This procedure is used to complement colposcopic examination; it allows observations of the endocervical canal and sees injuries that occur along its entire extension, and the entire squamocolumnar junction. This technique can provide an effective means to increase the sensitivity of colposcopy to detect lesions.

Keywords: Endocervix, Endocervicoscopy, Endocervicalcolposcopy, Endocervical mucosa, Cervical intraepithelial neoplasia, Colposcopy
Volume 9 Issue I - 2018

Isaac Melo

Oncology State Center, Mexico

Correspondence: Isaac Melo MD, Oncology State Center, Av. Rio San Miguel No. 10 Esq. Reforma, Colonia Proyecto Rio Sonora, C.P. 83250, Jazm?n de la Monta?a No. 2, Colonia Lomas Pitic, Hermosillo, Sonora, Mexico, C.P. 83010,Tel 52-662 2145316/2171669/2126916. Email Imelocl@live.com

Received: October 07, 2017 | Published: January 19, 2018

\section{Introduction}

In recent years, the diagnostic capacity of colposcopy has been called into question. Recent studies have indicated that the sensitivity of this method for the detection of cervical intraepithelial neoplasia 3 (CIN 3 ) is between $54 \%$ and $85 \% .{ }^{1}$ In additional, it has been reported that biopsies performed under colposcopy guidance detects only twothirds of CIN 3 lesions, ${ }^{2}$ and several researchers have even suggested that biopsies should be conducted randomly to improve their capacity to detect lesions. ${ }^{3,4}$

The exploration of the endocervix is necessary to study squamous and glandular cervical lesions. The following research findings highlight the importance of effective endocervical explorations:

a. Unsatisfactory colposcopy is the principal antecedent in many cases of persistent lesions ${ }^{5}$ and hidden invasive cancer. ${ }^{6,7}$

b. The squamocolumnar junction can be located as far as $2.5 \mathrm{~cm}$ within the endocervical canal. ${ }^{8}$

c. When a lesion penetrates the endocervical canal, the depth of the excision is critical for achieving proper treatment. ${ }^{9}$

d. The incidence of cervical adenocarcinoma is on the rise,$^{10}$ and this malignity frequently goes undetected by cytology, endocervical curettage, and colposcopy. ${ }^{11}$

There are multiple methods to explore the endocervical canal that can increase the efficacy of colposcopies. These range from the use of medications as misoprostol and estrogen, ${ }^{12,13}$ the use of endocervical specula of Menkel and Kogan, ${ }^{14}$ to the use of the microcolpohysteroscopy of Hamou. ${ }^{15}$ However, none of these methods has been shown to be ideal for properly observing and easily detecting lesions within the endocervical canal.

Brushing or Curettage of the endocervical canal ${ }^{16,17}$ is currently recommended as an ancillary procedure for unsatisfactory colposcopies, and in case of positivity it is recommended to proceed with a diagnostic loop electrosurgical excision procedure (LEEP) or cervical conization. ${ }^{18}$ However, the high percentage of false positives and negatives that are obtained using brushing or curettage, exposes patients to unnecessary surgical risks and often results in squeals that compromise the obstetrical future of the patients. ${ }^{19,20}$

Since the $80 \mathrm{~s}$, hysteroscopy have been used to observe the endocervical canal, ${ }^{21}$ but it was not until 2004 that Dr. Sergio Izzo $^{22}$ described his experience with 400 endocervicoscopy procedures in which he used a hysteroscope to observe the endocervical canal, this procedure is performed after the application of acetic acid to the interior of the endocervical canal, a technique that was later modified by Giuseppe Bifulco. ${ }^{23}$

In recent years, advances in illumination, optics, and video ${ }^{24}$ in combination with new exploratory techniques that do not require cervical dilatation, clamping, specula, or local anesthesia ${ }^{25}$ have allowed for the development of the office hysteroscopy, end the percentage of clinicians offering and practicing routinely this procedure it's increasing. 
The purpose of this study is to assess a novel technique for explore the endocervical canal, which is performed as a complement to colposcopy and enable more comprehensive explorations of the endocervical canal, thereby transforming unsatisfactory colposcopies into satisfactory colposcopies. This new technique allows detailed examinations of the endocervical canal and enables observations of the entire squamocolumnar junction, lesions that penetrate the canal and lesions that are found in its interior. This method, which we refer as endocervicalcolposcopy (ENCC), can be performed in the office and works in a similar fashion as colposcopy using direct applications of acetic acid to enhance contrast and make lesions more evident.

\section{Materials and methods}

Retrospective and Descriptive study, made from January 5, 2010 to June 30, 2014, conducted at the Clinic for Colposcopy of the State Oncology Center in Hermosillo City of Sonora State in Mexico. Any patient with abnormal cervical cytology was explored with Colposcopy and the exploration was complemented with Endocervicalcolposcopy. A follow-up period of at least two years to detect the persistence or recurrence of lesions and to rule out the existence of lesions that were not detected by either of the two methods. We have included all procedures that were conducted during the two first years, except for cases in which patients did not terminate their treatment or did not continue their follow-up for at least two years after diagnosis and treatment. Cases of Clinically invasive cancer were also excluded from our analysis. ENCCs were considered unsatisfactory when it was impossible to observe the entirety of the squamocolumnar junction, the entire extension of the lesion, or the endocervical canal before or after the application of acetic acid.

We began using the hysteroscope to explore the endocervical canal as a complementary technique during colposcopy explorations in 2008 . We have modified the technique described by Dr. Izzo by incorporating a technique that is similar to the procedure published by Dr. Giuseppe Bifulco. ${ }^{23}$ We use either hysteroscope a Bettochi or a Sopro Comeg hysteroscope with $4 \mathrm{~mm}$ operative sheath, a video camera with 3 chips, a Xenon illumination source, and a 500 cc bag of saline solution that is hung at a height of 2 meters (for gravity flow) as the distension medium. Immediately after conducting a standard colposcopy and recording the results of the colposcopy in digital form, we removed the vaginal speculum and began a hysteroscopic exploration with a "no-touch" (or vaginoscopy) approach. ${ }^{25}$ Next, we explore the exocervix and search for possible lesions along its entire extension. Using the iodine tincture (applied during the colposcopy), we clearly observed the squamocolumnar junction around its entire circumference and explore the endocervical canal up to the internal orifice (evaluating its characteristics that correspond to the physiological characteristics of the patient). Next, we remove the hysteroscope to the inside of the external orifice and we exchanged the distension medium for $3 \%$ acetic acid, which we inject via the same route that was use for the saline solution using a $10 \mathrm{cc}$ syringe and a solution extender to ensure that the flow of the solution is soft and continuous. We simultaneously explore the endocervical canal to detect any possible lesions and repeat our exploration of the squamocolumnar junction and the internal edge of the lesion. Biopsies are taken if any lesion is observed.

\section{Results}

Of the 260 colposcopies that were followed by ENCC, $124 \mathrm{did}$ not meet the inclusion criteria. Only 136 patients were fallow up. Among these colposcopy procedures, 79 patients $(58.08 \%)$ had satisfactory colposcopies (with average age of 32.57 years), and 57 patients $(41.91 \%)$ had unsatisfactory colposcopies (with average age of 42.51 years). Endocervicalcolposcopy detected 17 lesions (Table 1) that were not detected by colposcopy ( $12.5 \%$ of total patients), 14 of which had unsatisfactory colposcopy $(82.35 \%)$, and 3 had satisfactory colposcopy (17.64\%). A total of 8 ENCCs were not satisfactory (Table 1 ), and 4 had to be repeated but were satisfactory after a second exploration, in 3 of this patient's important lesions were founded: 1 cancer in situ, 1 well-differentiated adenocarcinoma, and 1 adenoma malignum also called minimal deviated adenocarcinoma. In the first two cases the ENCC was repeated because they presented abnormal cytology's 1 year after the first study, and the last one was repeated 1 month later of the first ENCC (after hormonal treatment because atrophy) because a glandular abnormality was suspected (due to the presence of crypts in the endocervical canal and abundant mucus secretion).

Table I 17 Lesions not detected by colposcopy

\begin{tabular}{|c|c|c|c|}
\hline $\begin{array}{l}\text { Lesions detected by } \\
\text { endocervicocolposcopy } \\
\text { and not detected by } \\
\text { colposcopy }\end{array}$ & $\begin{array}{l}\text { Satisfactory } \\
\text { colposcopy } \\
79(58.08 \%)\end{array}$ & $\begin{array}{l}\text { Unsatisfactory } \\
\text { colposcopy } 57 \\
(41.91 \%)\end{array}$ & $\begin{array}{l}\text { Total } 136 \\
100 \%\end{array}$ \\
\hline $\begin{array}{l}\text { Cervix with anatomical } \\
\text { alteration }\end{array}$ & 0 & I (I.75\%) & I (0.76\%) \\
\hline Lesion with deep penetration & & $4(7.01 \%)$ & $4(2.94 \%)$ \\
\hline Multifocal or jump lesion & $3(5.25 \%)$ & $6(10.25 \%)$ & $9(6.61 \%)$ \\
\hline $\begin{array}{l}\text { Other lesions (in the } 4 \\
\text { Repeated ENCCs) }\end{array}$ & 0 & $3(5.26 \%) * *$ & $3(2.20 \%) * *$ \\
\hline Total & $3(5.25 \%)$ & 14 (25.56\%) & $17(12.5 \%)$ \\
\hline Unsatisfactory ENCC & 0 & $8(14.03 \%) *$ & $8(5.88 \%)^{*}$ \\
\hline $\begin{array}{l}\text { Total unsatisfactory ENCC } \\
\text { with lesion }\end{array}$ & 0 & $3(37.5 \%)$ & $3(2.20 \%)$ \\
\hline
\end{tabular}

*of the 8 initially unsatisfactory ENCCs, 4 were repeated and became satisfactory; in these, ${ }^{* *}$ showed lesions.

The ENCC transformed 53 of the 57 unsatisfactory colposcopies (92.98\%) into satisfactory colposcopies (Graph 1). This technique permitted observations of the squamocolumnar junction, the entire extent of the lesion, and the endocervical canal. The method also allowed observations of the entire cervix of a patient with forced uterine retroversion (which prevented observations of the cervical orifice) and with repeated cytology's of a high grade lesion, which had been classified clinically as invasive cancer by the characteristics observed during exploratory. An ENCC didn't show data of invasion and a biopsy was performed that reported cancer in situ. Within the same group, 4 (7.01\%) patients had lesions with deep penetration that required an extension toward the endocervical canal of the process of excision to guarantee their complete excision.

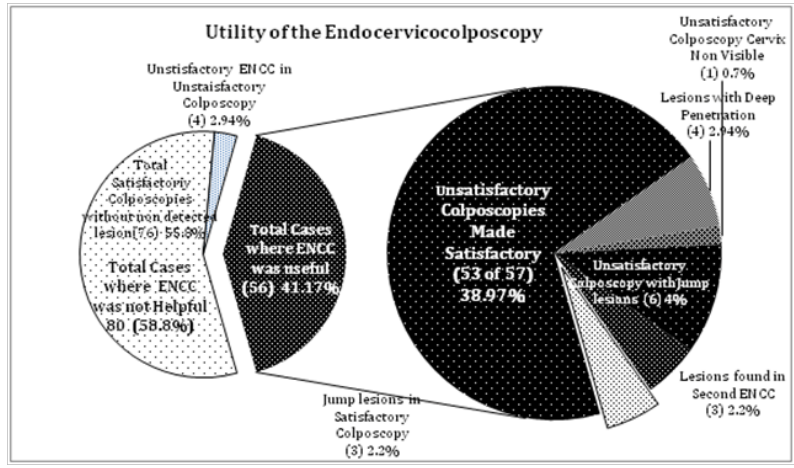

Graph I Utility of the Endocervicocolposcopy. 
I found $9(6.6 \%)$ multifocal or "jump lesions" which are lesions that have multiple foci end may set different grade penetrating the endocervical canal and can only be detected by this method, 6 in the group of unsatisfactory colposcopy and 3 in the group of satisfactory colposcopy. In the group of unsatisfactory colposcopy there were $3(2.16 \%)$ other lesions ( 2 of which were adenocarcinomas) that observed in the second ENCC. All this cases make a total of 56 in which ENCC was useful representing $41.17 \%$, and a $2.94 \%$ of failure rate (due to the 4 cases of unsatisfactory results obtained using this technique).

Table 2 Lesion persistence

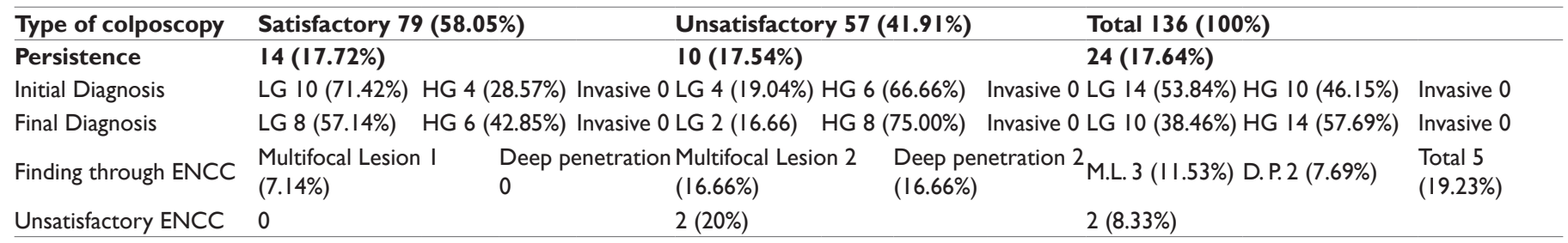

We observed 1 false positive case that occurred in a patient with a recent previous excisional surgery. The false positive resulted from the observation of an irregular mosaic pattern and punctuation resulting of the greater magnification from the proximity of the lens of the hysteroscope, this image was confused with a lesion. The biopsy reported metaplasia at the squamocolumnar junction (Figure 1).

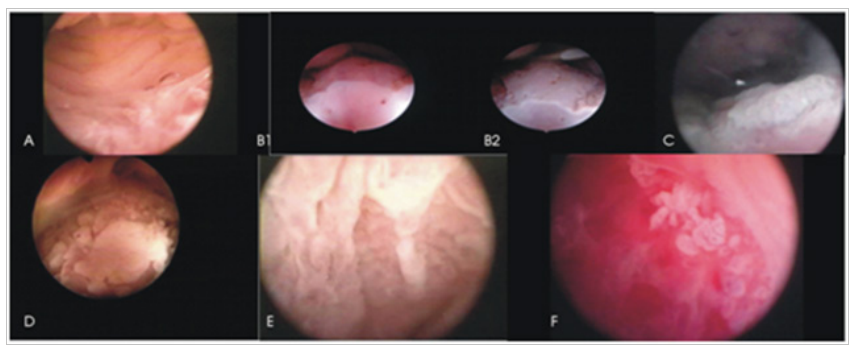

Figure I A: Normal endocervical canal, $B_{1}$ : Squamocolumnar junction at the beginning of the ENCC, $B_{2}$ : Squamocolumnar junction after the application of acetic acid, which reveals a lesion, C \& D: Multifocal or “jump lesions”, E: Lesion with deep penetration, F:Adenocarcinoma.

\section{Commentary}

ENCC is a simple exploratory method that is relatively easy to perform in the office and is used as a complementary ancillary procedure to standard colposcopy explorations. Using this method, we detected $12.5 \%$ of lesions that went undetected by colposcopy. The application of this novel technique allowed the observation and localization of lesions within the endocervical canal and estimations of the extent of these lesions. This procedure can help to ensure that proper therapeutic measures are taken for the complete removal of lesions diagnosed by biopsy. Furthermore, the use of this technique only prolongs the duration of exploratory colposcopy procedures by 5 to 10 minutes.

ENCC is not, by itself, a diagnostic method. Rather, ENCC is an exploratory method that facilitates the detection of lesions, which can be subsequently diagnosed by biopsies.

We detected 9 (6.61\%) multifocal or jump lesions that only can be detectable by ENCC. Among these lesions, 6 (66.66\%) occurred in patients with a history of previous treatment for mild dysplasia, which could be the source of their origin. Given that low-grade lesions typically receive excessive treatment in our country, a higher
All cases had follow-up a period of at least 2 years to verify the persistence or recurrence of lesions and to detect lesions that went undetected by either of the 2 exploratory methods. Table 2 shows the persistence of lesions during this period being in total 24 cases $(17.64 \%), 14(17.72 \%)$ of which were observed in the satisfactory colposcopy group, and 10 cases $(17.54 \%)$ of which were detected in the unsatisfactory colposcopy group. These results are consistent with reports from other authors. ${ }^{26-28}$

incidence of these types of lesions might be expected. Among these lesions, $8(88.88 \%)$ were high-grade lesions, and 1 was a microinvasive lesion (11.11\%). Furthermore, we detected 4 (2.94\%) lesions with deep penetrations. In these cases, a deeper excision was performed in an effort to reduce lesion persistence. We observed 2 early-stage adenocarcinomas and a rare case of adenoma malignum or minimum-deviation adenocarcinoma, none of which was detected by the colposcopy.

For each set of 4 unsatisfactory colposcopies (14 out of the 57 unsatisfactory colposcopies $25.56 \%$ ), a single lesion not detected by colposcopy (or a lesion with deep penetration) was observed. This result demonstrates the advantage of ENCC in improving the diagnostic capacity of colposcopy. The cases that were detected through ENCC may explain why an equal percentage of persistence was observed in the satisfactory and unsatisfactory colposcopy groups [14 (17.72\%) and $10(17.54 \%)$, respectively].

In relation of the 4 unsatisfactory ENCCs that were observed in this study (2.94\%), we have been able to resolve the visual obstruction produced by the cervical mucus. This solution is actually achieved through the application of a mucolytic agent (acetylcysteine), which is applied in a manner similar to that by which acetic acid is applied.

Overall, we can conclude that there is no justification for performing subsequent ENCCs in cases of satisfactory colposcopies because only 3 lesions in this group were undetected by colposcopy $(2.2 \%)$ and it was in this group that the false positive case appeared $(0.73 \%)$. These results are in agreement with previous reports suggesting that brush or curettage of the endocervical canal is unnecessary in cases of satisfactory colposcopies. ${ }^{19,29}$ However, an ENCC may be justified in cases with an antecedent of previous ablative or excisional treatment.

ENCC is an ideal complementary method for colposcopy in cases where cytology reveals glandular alterations because it allows for detailed explorations of the endocervical canal and uterine cavity. Is important avoid the temptation to explore the uterine cavity routinely because it is possible to only see a few benign of questionable clinical relevance findings and also exposes patients to surgical complications like Ott J reports, ${ }^{30}$ and a high possibility of bleeding that prevent a good observation.

It appears that observation of the endocervix is as important as observation of the exocervix, and ENCC may be the most appropriate 
and practical way to observe the endocervical canal, lesions along its entire extension, and the entire squamocolumnar junction.

ENCC should be used as a complementary ancillary procedure to colposcopy when the colposcopy is unsatisfactory and in cases in which the cytology indicate a potential glandular abnormality.

\section{Acknowledgements}

None.

\section{Conflicts of Interest}

None.

\section{References}

1. Cox JT More Questions About the Accuracy of Colposcopy What Does This Mean for Cervical Cancer Prevention? Obstet Gynecol. 2008;111(6):1266-1267

2. Gage JC, Hanson VW, Abbey K, et al.Number of Cervical Biopsies and Sensitivity of Colposcopy. Obstet Gynecol. 2006;108(2):254-272.

3. Nam K, Chung S, Kwak J, et al. Random Biopsy After ColposcopyDirect Biopsy Improves the Diagnostic of Cervical Intraepithelial Neoplasia Grade 2 or Worse. J Low Genital Tract Dis. 2010;14(4):346351

4. Pretorius RG, Belinson JL, Azizi F, et al. Utility of Random Cervical Biopsy and Endocervical Curettage in a Low-Risk Population. J Low Genital Tract Dis. 2010;16(4):333-338.

5. Hamontri S, Israngura N, Rochanawutanon M, et al. Predictive factors for disease in the uterine cervix after loop excision of the transformation zone in patients with cervical intraepithelial neoplasia III. J Med Assoc Thai 93 Suppl. 2010;2:S74-S80.

6. Kietpeerakool C, Sukkawattananon W, Srisomboon J, et al. Factors Predicting Occult Invasive Carcinoma in Women Undergoing a "See and treat" Approach. Asian Pacific J Cancer Prev. 2008;9(2):209-212.

7. Chen RJ, Chang DY, Yen ML, et al. Independent Clinical Factors Which Correlate with Failures in Diagnosing Early Cervical Cancer. Gynecol Oncol. 1995;58(3):356-361.

8. Parker MF, O Connor DM Measurement of Endocervical Canal Extension of the Transformation Zone on Fresh Hysterectomy Specimens. J Low Genit Tract Dis. 2003;7(4):254-258.

9. Ghaem-Maghami S, De-Silva D, Tipples M, et al. Determinants of Success in treating cervical intraepithelial neoplasia. BJOG. 2011;118(6):679-684.

10. Zaino RJ Glandular lesions of the uterine cervix. Mod Pathol. 2000;13(3):261-274.

11. Kietpeerakool C, Srisomboon J, Prompittayarat W, et al. Can adenocarcinoma in situ of the uterine cervix be predicted before cervical conization? Asian Pac J Cancer Prev. 2006;7(4):522-524.

12. Ayensu-Coker L, Sanchez J, Zurawin R, et al. Use of Misoprostol for Management of Unsatisfactory Colposcopy in the Adolecent: A Case Report and Review of the Literature. J Pediatr Adolesc Gynecol. 2009;22(5):e139-e141.
13. Piccoli R, Mandato VD, Lavitola G, et al. Atypical squamous cells and low squamous intraepithelial lesions in postmenopausal woman: Implications for management. Eur $J$ Obstet Gynecol Reprod Biol 2008;140(2):269-274.

14. Mossetti C Colposcopy yesterday and today. In: De Palo (Ed.), Colposcopy and Pathology of lower genital tract, Masson Spa, Milano, Italy, $p .1993 ; 51-77$

15. Hamou JE Microhysteroscopy, Clin Obstet Gynecol. 1983;26(2):285301.

16. Wright TC, Massad LS, Dunton CJ, et al. Consensus Guidelines for the Management of Woman with Cervical Intraepithelial Neoplasia or Adenocarcinoma In Situ. J Low Genit Tract Dis. . 2006 ;2007;11(4):223239

17. Zahn CM, Rao LK, Olsen C, et al. Reproducibility of Endocervical Curettage Diagnoses. Obstet Gynecol. 2011;118(2 Pt 1):240-248.

18. Krebs HB, Wheelock JB, Hurt WG. Positive Endocervical Curettage in Patients with Satisfactory and Unsatisfactory Colposcopy: Clinical Implications. Obstet Gynecol. 1987;69(4):601-605.

19. Spirtos NM, Schlaerth JB, d Ablaing G, et al. A Critical Evaluation of the Endocervical Curettage. Obstet Gynecol. 1987;70(5):729-733.

20. Larsson G. Outcome of pregnancy after conization. Acta Obstet Gynecol Scand. 1982;61(5):461-466.

21. Montevecchi L, Vecchione A. Endocervicoscopy. In: De Palo (Ed.), Colposcopy and Pathology of lower genital tract, Masson Spa, Milano, Italy. 1993;pp.301-305.

22. Izzo S, Negretti S, Stanco D, et al. The Endocervicoscopy. La Colposcopia in Italia Anno. 2004;XIX(3):13-20.

23. Bifulco G, Piccoli R, Lavitola G, et al. Endocervicoscopy: a new technique for the diagnostic work-up of cervical intraepithelial neoplasia allowing a tailored excisional therapy in Young fertile women. Fertil Steril. 2010;94(7):2726-2731.

24. Campo R, Van Belle Y, Rombauts L, et al. Office mini-hysteroscopy. Hum Reprod Update. 1999;5(1):73-81.

25. Van Dongen H, de Kroon CD, van den Tillaart SA, et al. A randomized comparison of vaginoscopic office hysteroscopy and saline infusion sonography: a patient compliance study. BJOG. 2008;115(10):12321237.

26. Lubrano A, Medina N, Benito V, et al. Follow-up after LLETZ: a study of 682 cases of CIN2-CIN3 in a single institution. Eur J Obstet Gynecol Reprod Biol. 2012;161(1):71-74.

27. Hellberg D, Nilsson S, Valentin J. Positive cervical smear with subsequent normal colposcopy and histology frequency of CIN in a long term follow-up. Gynecol Oncol. 199453(2):141-151.

28. Lu CH, Liu FS, Kuo CJ, et al. Prediction of Persistence or Recurrence After Conization for Cervical Intraepithelial Neoplasia III. Obstet Gynecol. 2006;107(4):830-835.

29. Lambert B, Lepage Y. Endocervical Curettage in Cervical Evaluation. $J$ Low Genit Tract Dis. 2000;4(3):125-127.

30. Ott J, Jatzko B, Nemeth Z, et al. Is routine hysteroscopy during LLETZ a valuable additional procedure? Acta Obstet Gynecol Scand. 2011;90(11):1284-1286. 